\title{
An Adaptive Scheme Based on Distributed Kalman Filter for Sensor Network
}

\author{
Zhang Kezhi \\ shanghai aircraft design and research institute
}

\begin{abstract}
Aiming at reducing the kinematic model error and uncertain measurement noise in sensor network, an adaptive scheme for distributed Kalman filter (DKF) is proposed in this paper. An adaptive factor is firstly applied to the covariance matrix of the predicted state vector to make the covariance estimation agree with its theoretical one, thus eliminating the effect of kinematic model error. Based on the innovation covariance of measurement noise and an adjustable fading factor, the other adaptive strategy is further developed for the updating of the covariance matrix of the measurement noise, which contributes to reduce the effect of the uncertain measurement noise. As demonstrated in simulation results, the adaptive distributed Kalman filter (ADKF) for sensor network performs better than the plain DKF in the case of suffering the kinematic model error and uncertain measurement noise.
\end{abstract}

Keywords-Adaptive distributed Kalman filter, sensor network, consensus filters

\section{INTRODUCTION}

Due to the ability of simple information processing, rapid deployment, self-organization and tolerance fault, sensor network technology has attracts wide attentions in environment monitoring, remote health care and mobile vehicles [1 2]. A key issue for sensor network is to develop effective methods to fuse the information generated by large-scale sensor networks [3]. At present the consensusbased information processing has been applied to sensor fusion in sensor networks [4 7]. Typically in the literature [7] a distributed Kalman filter is proposed which is reduced to two separate dynamic consensus problems in terms of weighted measurements and inverse-covariance matrices. By using low-pass and band-pass consensus filters, the data fusion can be solved. In the plain DKF algorithm it is supposed that the kinematic model can be built accurately and the statistical knowledge of measurement noise is also well known, thus guaranteeing the accuracy of state estimation. Unfortunately, due to the unknown operating environment, the fixed parameters of kinematic model may become unsuitable, and the statistical knowledge of measurement noise can also be uncertain, thereby leading to the inaccurate state estimation of sensor network. For eliminating the adverse effect of kinematic model error and the uncertain statistical knowledge of measurement noise, an adaptive scheme for DKF is to be developed.

In this paper some Kalman-filter -based adaptive methods have been investigated[8 11].In the above adaptive methods either the kinematic model error or the uncertain covariance matrix of measurement noise is discussed, but both of them have not been investigated simultaneously. Considering the problem, our objective is to build a DKF- based adaptive scheme for sensor network. An adaptive factor is firstly employed to the covariance matrix of the predicted state, which contributes to eliminate the effect of kinematic model error. The other adaptive strategy is further developed for the uncertain statistical knowledge of measurement noise. Then the two adaptive measures is employed to micro Kalman filter embedded in each sensor node, where the optimal estimation of the state can be obtained, thereby improving the estimation accuracy of sensor network in the unknown operating environment.

\section{ADAPTIVE SCHEME BASED ON DKF}

Due to the unknown operating environment, the parameters of the transition matrix $A_{i}(k+1 \mid k)$ can be disturbed, and the statistical knowledge of the process noise $Q_{i}$ also becomes uncertain, both of which constitute the kinematic model error. On the other hand, the measurement noise covariance $R_{i}$ is also uncertain. Therefore the state estimation by using the plain DKF can be not accurate, even divergent.

\section{A. Adaptive Factor for the Kinematic Model Error}

The objective is to modify the covariance matrix of the predicted state vector by introducing an adaptive factor, thus reducing the effect of kinematic model error.

Since the disturbed transition matrix and uncertain statistical knowledge of the process noise have an obvious effect on the covariance matrix of the predicted state, therefore an adaptive factor can be applied to the modification of the covariance matrix of the predicted state, thus eliminating the effect of the kinematic error.

It is assumed that the predicted residual vector is obtained using the available measurement vector $z_{i}(k)$ at epoch $k$

$$
r_{i}(k \mid k-1)=C_{i}(k) x(k \mid k-1)-z_{i}(k)
$$

From Eq.1 and the covariance propagation law, the theoretical one of the covariance matrix of the predicted residual vector can be obtained.

$$
P_{i}^{r 1}(k \mid k-1)=C_{i}(k) P_{i}(k \mid k-1) C_{i}^{T}(k)+R_{i}(k)
$$

To adapt the inaccurate kinematic model, the covariance matrix of the predicted state vector is to be changed into $\frac{1}{a_{i}(k)} P_{i}(k \mid k-1)$. Thereby the estimated covariance matrix of the predicted residual vector is written as 


$$
\bar{P}_{i}^{r 1}(k \mid k-1)=\frac{1}{a_{i}(k)} C_{i}(k) P_{i}(k \mid k-1) \cdot C_{i}^{T}(k)+R_{i}(k)
$$

Where $a_{i}(k)$ denotes the adaptive factor.

Assuming that the estimation of the covariance matrix of the predicted residual vector, denoted as $\hat{P}_{i}^{r 1}(k \mid k-1)$, equals the corresponding theoretical one $\bar{P}_{i}^{r 1}(k \mid k-1)$

$$
\widehat{P}_{i}^{r 1}(k \mid k-1)=\bar{P}_{i}^{r 1}(k \mid k-1)
$$

Based on Eq.4, it can be obtained

$$
\widehat{P}_{i}^{11}(k \mid k-1)=\frac{1}{a_{i}(k)} C_{i}(k) P_{i}(k \mid k-1) \cdot C_{i}^{T}(k)+R_{i}(k)(5)
$$

The left and right of Eq.5 are multiplied by $a_{i}(k)$

$$
a_{i}(k) \hat{P}_{i}^{\text {'1 }}(k \mid k-1)=C_{i}(k) P_{i}(k \mid k-1) \cdot C_{i}^{T}(k)+a_{i}(k) R_{i}(k)
$$

By using Eq.2 the further derivation of Eq. 6 can be written

$$
a_{i}(k) \hat{P}_{i}^{r 1}(k \mid k-1)=P_{i}^{r 1}(k \mid k-1)-R_{i}(k)+a_{i}(k) R_{i}(k)
$$

(7)

Thereby the adaptive factor of sensor node $i$ can be obtained

$$
a_{i}(k)=\frac{\operatorname{tr}\left(P_{i}^{r 1}(k \mid k-1)-R_{i}(k)\right)}{\operatorname{tr}\left(\widehat{P}_{i}^{r 1}(k \mid k-1)-R_{i}(k)\right)}
$$

$\operatorname{tr}()$ denotes the trace of the matrix. In practical applications, the adaptive factor is achieved $a_{i}(k) \leq 1$, therefore

$$
a_{i}(k)=\left\{\begin{array}{l}
1 ; \operatorname{tr}\left(P_{i}^{r 1}(k \mid k-1)\right)>\operatorname{tr}\left(\hat{P}_{i}^{r 1}(k \mid k-1)\right. \\
\frac{\operatorname{tr}\left(P_{i}^{r 1}(k \mid k-1)-R_{i}(k)\right)}{\operatorname{tr}\left(\widehat{P}_{i}^{r 1}(k \mid k-1)-R_{i}(k)\right)} ; \text { otherwise }
\end{array}\right.
$$

To reflect the kinematic model error accurately, $\hat{P}_{i}^{r 1}(k \mid k-1)$ is to be estimated using the predicted residual vector at the present epoch

$$
\widehat{P}_{i}^{r 1}(k \mid k-1)=r_{i}(k \mid k-1) r_{i}^{T}(k \mid k-1)
$$

\section{B. Adaptive Measurement Covariance for Uncertain} Measurement Noise

Although the effect of the kinematic model error is reduced by using the adaptive factor, it is not optimal because of uncertain statistical knowledge of measurement noise.

Considering the measurement noise is statistically independent from the kinematic model error, we propose an adaptive strategy for uncertain statistical knowledge of measurement noise. In the approach the measurement difference in fixed time window is firstly employed to evaluate the innovation covariance of measurement noise.
It is assumed that the first order and second order difference for the measurements are, respectively, denoted by Eq. 11 and Eq. 12 .

$$
\begin{gathered}
z_{i}^{1}(k+1)=z_{i}(k+1)-z_{i}(k) \\
z_{i}^{2}(k+1)=z_{i}^{1}(k+1)-z_{i}^{1}(k)
\end{gathered}
$$

For the measurements in fixed time window, the innovation covariance of measurement noise can be written as

$$
\begin{array}{r}
R_{i}^{i n n}(k)=\frac{1}{M} \sum_{m=1}^{M}\left(z_{i}^{d}(m)-\bar{z}_{i}^{d}\right)\left(z_{i}^{d}(m)-\bar{z}_{i}^{d}\right)^{T} \\
\bar{z}_{i}^{d}=\frac{1}{M} \sum_{m=1}^{M} z_{i}^{d}(m)
\end{array}
$$

If the results using first order difference can be approximate to the white noise, the constant $d=1$ is to be selected, else the second order difference $d=2$ should be employed. $M$ is the size of time window. Generally using the larger size of time window can improve the evaluation accuracy of the innovation covariance of measurement noise, thus the optimal estimation of the state can be obtained.

Based on Eq.13, the covariance matrix of measurement noise can be updated

$$
R_{i}(k)=d_{i}(k) R_{i}(k-1)+\left(1-d_{i}(k)\right) R_{i}^{I n n}(k)
$$

Where $d_{i}(k)$ is denoted as

$$
\begin{cases}d_{i}(k)=\frac{1-b_{i}(k)}{1-b_{i}(k)^{k+1}} & 0 \leq b_{i}(k)<1 \\ d_{i}(k)=0 & b_{i}(k)=1\end{cases}
$$

Generally $b_{i}$, fading factor, is fixed as a constant [12]. With the increasing epoch, $d_{i}(k)$ will converge toward a constant. However, the fixed factor is not suitable in the case of uncertain measurement noise. Especially when the measurement noise becomes larger, the old of covariance matrix should play minor role in the updating of measurement noise covariance. In other words, the innovation covariance should be highlighted. Therefore the fading factor should be increased in the case. Here $b_{i}$ is modified as follows.

$$
\begin{aligned}
& \text { If } \operatorname{tr}\left(R_{i}(k)\right)>\operatorname{tr}\left(R_{i}(k-1)\right) \text { and } b(k) \leq 1, \\
& \qquad \begin{aligned}
b_{i}(k)=\frac{\operatorname{tr}\left(R_{i}(k)\right)}{\operatorname{tr}\left(R_{i}(k-1)\right)} \cdot b_{i}(k-1) \\
\text { Otherwise, } b_{i}(k)=b_{i}(k-1)
\end{aligned}
\end{aligned}
$$

Because of noise in the course measure, data from optical triangular scanners is preprocessed, which includes smoothing, removal noise and deleting exceptional points, before reconstructing surface is cable of identification clouds and process, the minimum distance is found automatically and tandem compound to acquire point group. Cut point group along a certain direction to obtain crosssection these are continuous and in a sequence [4]. 


\section{III. . SimUlation RESUltS AND DiscUSSIONS}

For validate the adaptive distributed Kalman filter (ADKF), three groups of numerical simulations are implemented. The first group is employed to validate the effect of ADKF in the case of the accurate kinematic model and little uncertainty of measurement noise, and the second group is used for the validation in the case of the accurate kinematic model and obvious uncertainty of measurement noise, and the third group is used for the validation in the case of inaccurate kinematic model and obvious uncertainty of measurement noise.

Their simulation results are showed in Figure1-3 and Table I.

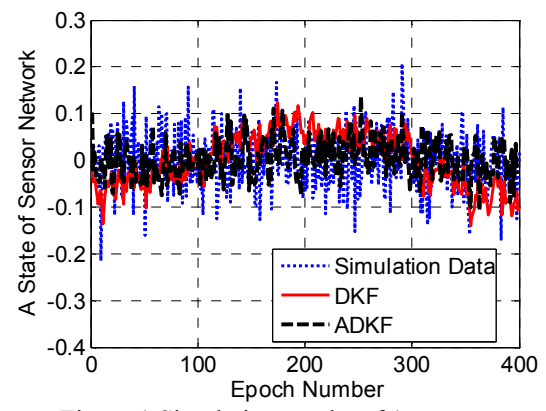

Figure 1 Simulation results of 1 st group

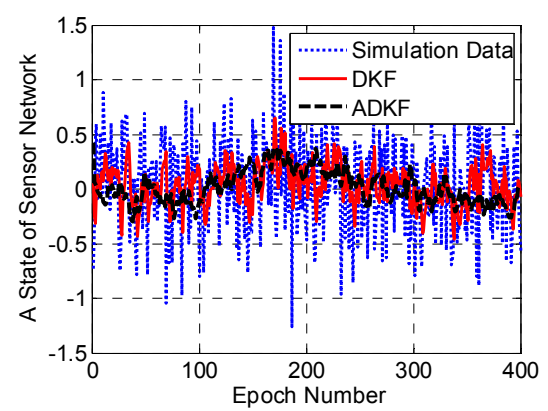

Figure 2 Simulation results of 2 sd group

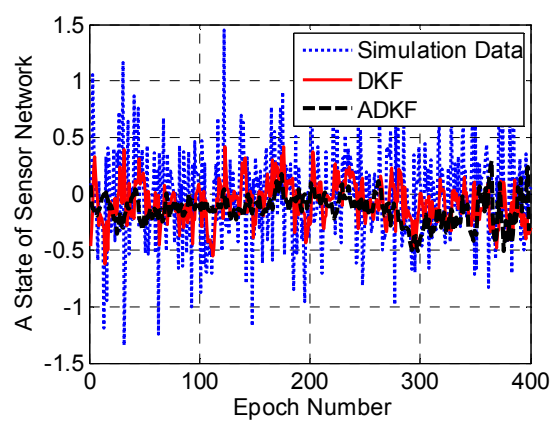

Figure 3 Simulation results of 3rd group
TABLE I STANDARD VARIANCE OF FILTERED RESIDUAL ERROR

\begin{tabular}{|c|c|c|c|}
\hline & $\begin{array}{l}\text { Simulation } \\
\text { Data }\end{array}$ & DKF & ADKF \\
\hline $\begin{array}{l}\text { Simulation } \\
\text { Group1 }\end{array}$ & $\begin{array}{ll} & 0.0624 \\
3 & \\
\end{array}$ & $\begin{array}{ll} & 0.0560 \\
1 & \\
\end{array}$ & $957^{0.03}$ \\
\hline $\begin{array}{l}\text { Simulation } \\
\text { Group2 }\end{array}$ & 0.4187 & 0.1841 & $91^{0.14}$ \\
\hline $\begin{array}{l}\text { Simulation } \\
\text { Group3 }\end{array}$ & 0.4138 & 0.1817 & 0.1285 \\
\hline
\end{tabular}

Based the above simulation results, it can be observed that the proposed adaptive strategy for DKF shows the better performance than the plain DKF in term of the standard variance of filtered residual error. That can be attributed to two facts:

1) An adaptive factor is applied to the covariance matrix of the predicted state vector to make the right hand of Eq.18 agree with its estimation, thus contributing to eliminating the effect of the kinematic model error.

2) The time-window-based innovation covariance of measurement noise is calculated in Eq.13, and then the adjustable fading factor is further employed to the updating of measurement noise covariance Eq.15, thereby the effect of uncertain statistical knowledge can be greatly eliminated

\section{SUMMARIES}

Considering the kinematic model error and uncertain statistical knowledge of measurement noise resulted from the operating environment, we propose a DKF-based adaptive scheme for sensor network, where two adaptive mechanisms are introduced to the micro Kalman filter embedded in sensor node. From the simulation results, it can be observed that ADKF by employing the two adaptive measures perform better than DKF in the case of kinematic model error and uncertain statistical knowledge of measurement noise, thus the accurate state estimation of sensor node can be obtained. Accordingly ADKF can be a promising method for data fusion of sensor network. In the further work, some field experiments will be done to validate the proposed algorithm.

\section{REFERENCES}

[1] I. Akyildiz, W. Su, Y. Sankarasubramniam, and E. Cayirci, "A survey on sensor networks," IEEE Communications Magazine, , August 2002, PP.102-114.

[2] J. Cortes, S. Martinez, T. Karatas, and F. Bullo, "Coverage control for mobile sensing networks," The IEEE Trans. on Robotics and Automation, vol.20, no.2, April 2004, PP.243-255.

[3] H. Durrant-Whyte, "Data fusion in sensor networks," Fourth International Symposium on Information Processing in Sensor Networks, April 2005.

[4] R. Olfati-Saber and R. M. Murray, "Consensus problems in networks of agents with switching topology and time-delayss," IEEE Trans. on Automatic Control, vol. 49, no. 9, September 2004, PP. 1520-1533.

[5] W. Ren and R. W. Beard, "Multi-agent consensus with relative uncertainty," American Control Conference, Portland, June 2005.

[6] D. B. Kinfston and W. Ren, "Consensus algorithm are input-to-state stable," American Control Conference, Portland, June 2005. 
[7] R. Olfati-Saber. "Distributed Kalman Filter with Embedded Consensus Filters," Proc. of the Joint CDC-ECC '05 Conference, December 2005.

[8] J. Wang et al., "Online Stochastic Modeling for network-based GPS real-time kinematic positioning," Journal of Global Positioning Systems, vol.1, no. 4, pp. 113-119, February 2005.

[9] A. H. Mohamed, K. P. Schwarz, "Adaptive Kalman filtering for INS/GPS," Journal of Geodesy, vol.73, no.4, May 1999, PP. 193-203.

[10] N.A.White, P.S.Maybeck, S.L.DeVilbiss, "Detection of interference/jamming and spoofing in a DGPS-aided inertial system," Aerospace and Electronic Systems IEEE Transactions on, vol.34,no.4, October 1998, PP. 1208 - 1217.

[11] P.S.Maybeck, M.R.Schore, "Robustness of a moving-bank multiple model adaptive algorithm for control of a flexible space structure," Aerospace and Electronics Conference, 1990. NAECON 1990, Proceedings of the IEEE 1990 National, vol.1, no.1, May 1990, PP. $368-374$.

[12] Taewon Yang, JooHun Lee, Ki Yong Lee and Koeng-Mo Sung. "On robust Kalman filtering with forgetting factor for sequential speech analysis,” Signal Processing, Vo.63,no.2, 1997, PP.151-156. 